\title{
Multiclass Utility-Based Scheduling for UWB Networks
}

\author{
Kuang-Hao Liu, Lin Cai, Member, IEEE, and Xuemin Shen, Senior Member, IEEE
}

\begin{abstract}
In this paper, the optimal scheduling problem for ultrawideband (UWB) networks is formulated as a utility maximization problem, considering heterogeneous traffic characteristics and the fairness constraint, which is an NP-hard problem. The utility function, defined as the user satisfaction level with respect to the allocated bandwidth, takes the link distance as the input parameter that may be noisy due to ranging errors. We solve the stochastic optimization problem by a metaheuristic, called the exclusive-region-based global search algorithm, that can avoid trapping into local optima with reasonable efficiency, complexity, and convergence speed. The concept of exclusive region for concurrent UWB communications is employed to improve algorithm efficiency. Extensive simulations demonstrate that, by appropriately scheduling concurrent transmissions, the total utilities of each traffic class can be significantly improved. In addition, the proposed scheduling algorithm can support heterogeneous applications and guarantee the intraclass and interclass fairness among competing flows.
\end{abstract}

Index Terms-Discrete stochastic optimization, medium access control (MAC), scheduling, ultrawideband (UWB), utility function.

\section{INTRODUCTION}

$\mathbf{U}$ LTRAWIDEBAND (UWB) is a promising technology for future wireless personal area networks (WPANs). UWB devices can exchange high-volume multimedia traffic or deliver high-data-rate traffic to/from the Internet [1], [2]. In IEEE 802.15.3-based WPANs, an autonomously selected network coordinator, called the piconet controller (PNC), allocates wireless resources (i.e., time slots) according to users' quality-ofservice (QoS) requirements in a time-division multiple-access (TDMA) fashion [3]. During the allocated time slot, devices can communicate in a peer-to-peer mode. The IEEE 802.15.3 medium access control (MAC) protocol was originally proposed for narrowband wireless communications; hence, it is not optimal for UWB networks. In addition, the distinct characteristics of UWB, such as concurrent transmissions and ranging capability, have not been considered in the MAC protocol design.

Manuscript received November 16, 2006; revised May 4, 2007 and June 14, 2007. This paper was presented in part at the IEEE Consumer Communications and Networking Conference, Las Vegas, NV, January 2007. The review of this paper was coordinated by Dr. Q. Zhang.

K.-H. Liu and X. Shen are with the Centre for Wireless Communications, Department of Electrical and Computer Engineering, University of Waterloo, Waterloo, ON N2L 3G1, Canada (e-mail: k8liu@bbcr.uwaterloo.ca; xshen@bbcr.uwaterloo.ca).

L. Cai is with the Department of Electrical and Computer Engineering, University of Victoria, Victoria, BC V8W 3P6, Canada (e-mail: cai@ece.uvic.ca).

Color versions of one or more of the figures in this paper are available online at http://ieeexplore.iee.org.

Digital Object Identifier 10.1109/TVT.2007.906378
Since the very first impulse-radio UWB, several variations of UWB systems, such as impulse-based direct-sequence UWB [4] and multiband (MB) orthogonal frequency-division multiplexing [5]-based UWB, have been proposed. Although these UWB systems employ different techniques to spread the signal over the wide spectrum, their common feature is the capability of allowing concurrent transmissions. From an individual user's aspect, concurrent transmissions in the vicinity degrade the received signal quality and, thus, the instantaneous data rate due to the increased multiple-user interference (MUI). On the other hand, by allowing concurrent transmissions, each user may be allocated more transmission time slots. Consequently, their total throughput can be improved if the interference is appropriately controlled.

UWB-based WPANs are anticipated to support heterogeneous traffic such as real-time video/audio streaming and bulk file transfer. Concerning efficient and fair resource allocation, utility is an effective measure of QoS [7]-[10]. Utility reflects the user's satisfaction level to the assigned resource. Moreover, considerations such as price or differentiated services can be flexibly incorporated into the utility functions [11], [12]. Considering the heterogeneous traffic characteristics, in this paper, we formulate the optimal scheduling problem as a utility maximization problem. A utility-based scheduling algorithm aiming at multiclass QoS provisioning with fairness consideration is proposed. Generally, an efficient scheduling algorithm requires feedback information from the network to appropriately make scheduling decisions [13]. When all UWB devices communicate in a peer-to-peer manner, it is very difficult, if not impossible, for the PNC to acquire instantaneous channel information of each flow. To estimate the achievable data rate of the target flow, the PNC can make use of the ranging capability featured by UWB communications [14], [15]. However, the distance information obtained may be noisy due to multipath fading. Since the objective function is evaluated based on the measured distance information, the utility estimation may be biased, and thus affects the scheduling decisions. When the objective function of the optimization problem contains uncertainty, the problem becomes stochastic. Since the analysis of the noisy objective function is not tractable in our case, we resort to metaheuristic methods and choose to use the global search algorithm (GSA) [17]. This is because its convergence to the global optimum can be proved, and the tradeoff between computational complexity and efficiency is tunable. We tailor the GSA for the UWB scheduling problem and show that the modified algorithm, called the exclusive-region-based GSA (ER-GSA), has a desired convergence with reasonable computational complexity for practical implementations. 


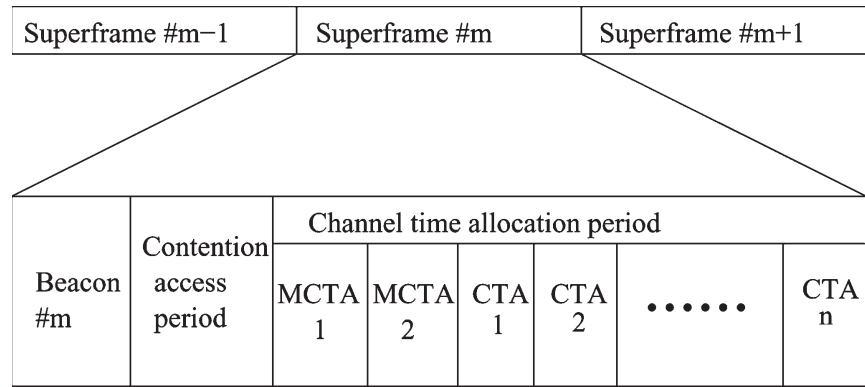

MCTA: Management Channel Time Allocations

CTA: Channel Time Allocations

Fig. 1. Superframe structure defined in the IEEE 802.15.3 MAC protocol.

The main contributions of this paper are threefold: 1) The scheduling for concurrent UWB transmissions that maximizes the weighted utility is formulated, which is an NP-hard problem; 2) a utility-based scheduling scheme is proposed to support multiclass traffic with fairness constraint; and 3) the assumption of perfect distance information for measuring flow throughput is relaxed by factoring estimation errors into the objective function. The stochastic optimization problem is solved by the proposed ER-GSA, and its convergence property and computational complexity are studied.

The remainder of this paper is organized as follows: Section II describes the system model and characterizes three traffic classes by different utility functions. In Section III, we first derive the sufficient condition that concurrent UWB transmission schemes are favorable to TDMA. Based on that, we formulate the optimal scheduling problem for multiclass traffic. The ER-GSA algorithm for solving the stochastic optimization problem is presented in Section IV. Simulation results are given in Section V. The related work is summarized in Section VI, followed by the concluding remarks in Section VII.

\section{SySTEM MODEL}

\section{A. Network Structure and Channel Model}

Consider UWB-based WPANs using the IEEE 802.15.3 MAC protocol. According to the IEEE 802.15.3 standard, time is slotted into a superframe structure, as shown in Fig. 1, consisting of a beacon period (BP), a contention access period (CAP) using carrier sensing multiple access with collision avoidance (CSMA/CA), and a contention-free period, called the channel time allocation period (CTAP), using TDMA. One of the users, acting as the PNC, performs network management and allocates channel time slots in the CTAP. Each active user sends the channel time request through contentions in CAP and uses the allocated channel times in the CTAP for peer-to-peer transmissions, i.e., users can communicate with each other in the CTAP without the involvement of the PNC. Such a hybrid access mechanism is more efficient than polling-based resource allocation schemes and provides better QoS than random access schemes.

To estimate the data rate, we use the following simplified channel model: Assume that a UWB receiver can adapt its transmission rate to an arbitrary signal-to-interference-plusnoise-ratio (SINR) level. According to Shannon's theory, for a sufficiently large bandwidth, the achievable data rate $r_{i}$ of flow $i$ is upper bounded by $r_{i}=k p_{r}(i) /(I+\eta)$, where the coefficient $k$ is a constant, $p_{r}(i)$ is the received power at flow $i$ 's receiver, $\eta$ is the average power of the background noise, and $I$ is the interference power. The received power at the $i$ th receiver is $p_{r}(i)=\alpha p_{t}(i) d_{i}^{-\gamma}$, where $\alpha$ is reciprocal of the processing gain of UWB signals, $d_{i}$ is the distance between the transmitter and the receiver of flow $i$, and $\gamma$ is the pathloss exponent. Both parameters $\alpha$ and $\gamma$ are considered to be constant. Here, we neglect the multipath fast fading when we estimate the average data rate $r_{i}$.

\section{B. Utility Functions}

Utility is defined as the satisfaction level of a user with respect to the amount of allocated bandwidth. Various utility functions have been proposed to characterize different traffic types [7]-[10]. To deal with heterogeneous traffic types, the utility functions considered in this paper are general nondecreasing functions with values within $[0,1]$. Traffic types are classified into three classes. Class 1 includes constant bit-rate applications, for example, audio streams. We use a step function for Class 1, i.e.,

$$
u(r)= \begin{cases}1, & \text { if } r \geq r_{\min } \\ 0, & \text { if } r<r_{\text {min }}\end{cases}
$$

where $r_{\min }$ is the minimum bandwidth requirement for a connection. Class-2 applications can adapt to the allocated bandwidth to a certain extent, e.g., video stream, as described by the following sigmoidal-like utility function:

$$
u(r)=1-e^{-\frac{b r^{2}}{a+r}}
$$

where the parameters $a$ and $b$ can be adjusted to determine the shape of $u(r)$. Class-3 applications are most flexible to the available bandwidth. Most Transmission-Control-Protocolcontrolled data applications belong to this class. Since there is no minimum rate requirement for such traffic class, the utility can be modeled by the following function:

$$
u(r)= \begin{cases}1, & \text { if } r \geq r_{\max } \\ \sin ^{\tau}\left(\frac{\pi}{2} \cdot \frac{r}{r_{\max }}\right), & \text { if } r<r_{\max }\end{cases}
$$

where the parameter $\tau$ controls the shape of $u(r)$, as shown in Fig. 2(b). See [21] for more discussions on different utility functions. Generally, the choice of utility functions would affect the efficiency of resource allocation and introduce different degrees of complexity to the utility maximization problem [22].

\section{Optimal Scheduling With CONCURRENT TRANSMISSIONS}

In this section, we first derive the sufficient condition to ensure that concurrent transmissions result in a higher 


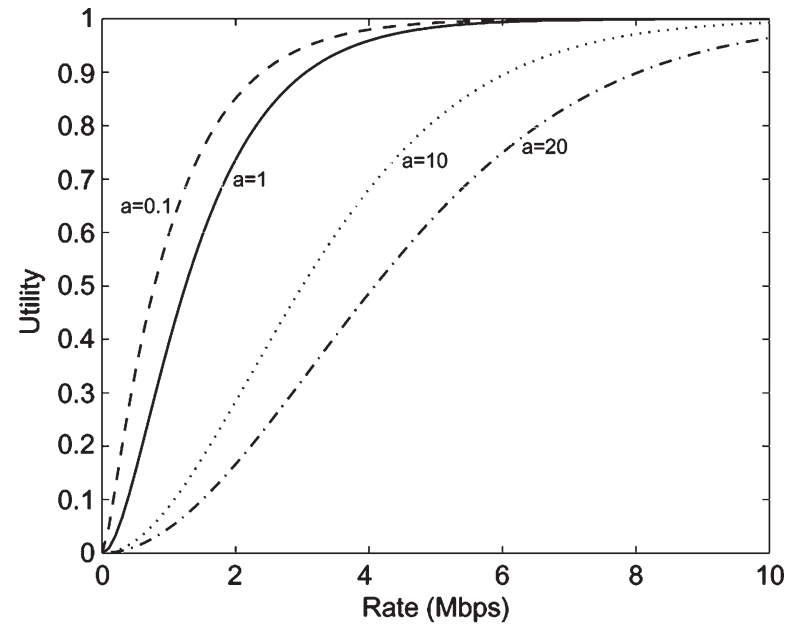

(a)

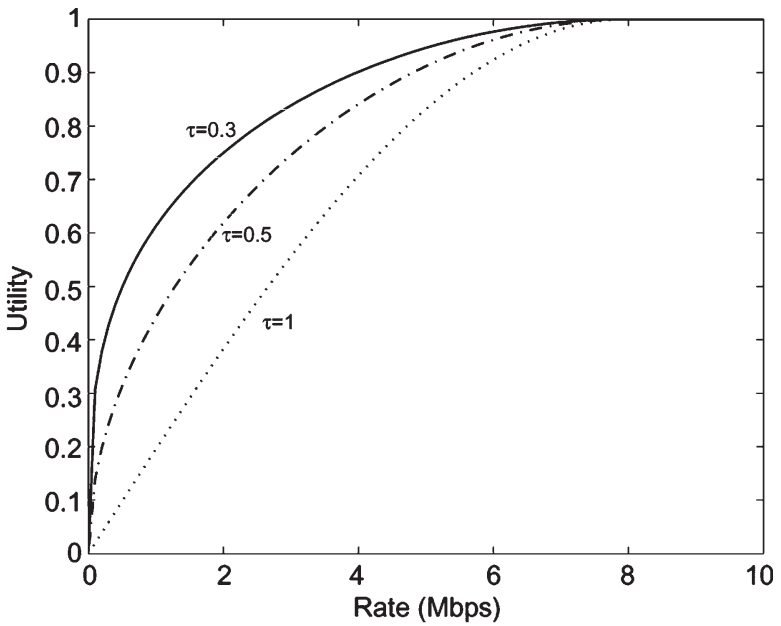

(b)

Fig. 2. Illustrative utility functions for Class- 2 and Class-3 traffic. (a) Utility functions for Class $2(b=1)$. (b) Utility functions for Class $3\left(r_{\max }=8 \mathrm{Mb} / \mathrm{s}\right)$.

aggregate throughput than TDMA. We then formulate the optimal scheduling problem with concurrent transmissions.

\section{A. Concurrent Transmissions}

For peer-to-peer UWB transmissions during the CTAP, the cross correlations of concurrent transmissions lead to MUI [23]. When MUI is present, the achievable date rate of a target flow is lower than that of without interference. Its cumulative throughput over multiple slots, however, can be increased by time-domain multiplexing if the concurrent transmission set is elaborately selected.

Consider $n$ users requesting transmissions in $n$ time slots. If $n$ requests are scheduled in a TDMA fashion, i.e., each slot is assigned to only one user, the achievable throughput during $n$ slots for the $i$ th user, denoted by $r_{i}^{T}$, can be represented by

$$
r_{i}^{T}=k p_{r}(i) /(n \eta)=\alpha k p_{t}(i) d_{i}^{-\gamma} /(n \eta) .
$$

On the other hand, concurrently scheduling $n$ flows for $n$ slots leads to the throughput of user $i r_{i}^{C}$

$$
r_{i}^{C}=\frac{k p_{r}(i)}{\eta+\sum_{j \neq i} I_{j, i}}=\frac{\alpha k p_{t}(i) d_{i}^{-\gamma}}{\eta+\sum_{j \neq i} I_{j, i}}
$$

where $I_{j, i}$ is the interference from the $j$ th sender to the $i$ th receiver, which is proportional to $d_{j, i}^{-\gamma}$. Denote $D$ the distance such that $I_{j, i}$ equals $\eta$. If all interferers are at least $D$ away from the receiver of the $i$ th flow (i.e., $d_{j, i} \geq D$ ), we have $I_{j, i} \leq \eta$ for all $j \neq i$. The aforementioned condition implies $r_{i}^{C} \geq \alpha k p_{t}(i) d_{i}^{-\gamma} /(\eta+(n-1) \eta)=\left(\alpha k p_{t}(i) d_{i}^{-\gamma} / n \eta\right)=r_{i}^{T}$. In other words, when each receiver maintains an exclusive region with a radius not less than $D$, in which no other transmitters are allowed to transmit, concurrent UWB communications are preferable to TDMA transmissions. Thus, any two flows $i$ and $j$ can concurrently transmit to produce a higher throughput if the following condition holds:

$$
d_{i, j}>D \quad \text { and } \quad d_{j, i}>D .
$$

Note that the exclusive region for UWB networks is independent of the distance between the sender-receiver. This is different from the protocol model considered in [6] for communications without rate adaptation.

\section{B. Optimal Scheduling}

To increase the total throughput, it is desirable to have concurrent transmissions if condition (6) holds. Therefore, the objective of scheduling is to select the optimal flow set for each time slot such that the total user utilities are maximized. Taking the MUI of concurrent transmissions into account, the network can be modeled as a conflict graph $G=(V, E)$, where the vertex set $V$ represents the set of flows requesting for transmission, and $E$ is the set of edges. Two vertices in $G$ are connected if they are not allowed to transmit in the same time slot according to the exclusive-region condition (6). The connected vertices (flows) are called neighbors. Let $N(i)$ represent the neighbors of a vertex $i \in V$. Define the following binary variables: $x_{i}^{s}=1$ if flow $i$ is allocated in slot $s$; otherwise, $x_{i}^{s}=$ 0. $y_{i j}^{s}=1$ if node $j$ is the recipient of node $i$ in slot $s$; otherwise, $y_{i j}^{s}=0$. Denote $U\left(r_{i}\right)$ the utility function of user $i$ with the achievable data rate $r_{i}$. The scheduling problem for a single slot can be formulated as a nonlinear mixed-integer optimization problem, i.e.,

$$
\begin{aligned}
& \text { (P) } \quad \max \sum_{i=1}^{|V|} x_{i}^{s} U\left(r_{i}\right) \\
& \text { s.t. } \quad x_{i}^{s} \sum_{j \in N(i)} x_{j}^{s}=0 \quad \forall i=1, \ldots,|V| \\
& \quad \sum_{i \in \mathcal{T}} y_{i j}^{s}+\sum_{k \in \mathcal{R}} y_{j k}^{s} \leq 1 \quad \forall j \in \mathcal{R} \\
& r_{i}=k p_{t} d_{i}^{-\gamma} /\left(\eta+x_{j}^{s} \alpha p_{t} \sum_{j=1, j \neq i}^{|V|} d_{j i}^{-\gamma}\right), \quad i=1, \ldots,|V|
\end{aligned}
$$


Given the number of active flows $|V|$ and the current slot $s$, the flow set $\left\{x_{i}^{s}: i=1, \ldots,|V|\right\}$, which maximizes the total utilities defined in (7) under the constraints of (8)-(10), is the optimum of $(\mathcal{P})$. Constraint (8) ensures that any flow scheduled in slot $s$ is conflict free. Constraint (9) ensures that a node can communicate with at most one node in a slot, where $\mathcal{T}$ represents the set of senders being scheduled in the same slot, and $\mathcal{R}$ is the set of corresponding receivers. Constraint (10) specifies the flow data rate. Note that $(\mathcal{P})$ can be extended as a superframe utility optimization problem by modifying (7) as $\max \sum_{s=1}^{S} \sum_{i=1}^{|V|} x_{i}^{s} U\left(r_{i}\right)$, where $S$ is the total number of slots in a superframe.

Problem $(\mathcal{P})$ can be reduced to the maximum weighted independent set (MWIS) ${ }^{1}$ problem, as interpreted in the following: We first incorporate constraint (9) into the graph $G$ by adding an edge between two nodes in $V$ if the corresponding flows have common transmitting or receiving nodes and then denote the resulting graph as $G^{\prime}$. Let $\mathcal{K}$ denote the collection of independent sets in $G^{\prime}$. Since the superset $\mathcal{K}$ satisfies the constraints (8) and (9), finding the optimal solution of problem $(\mathcal{P})$ is equivalent to finding the set with the maximum weight in $\mathcal{K}$, which is denoted by $\kappa^{*} \in \mathcal{K}$ in $G^{\prime}$. In other words

$$
\kappa^{*}=\arg \max _{\kappa \in \mathcal{K}} \sum_{i=1}^{|\kappa|} U\left(r_{i}\right)
$$

where $U\left(r_{i}\right)$ corresponds to the weight of vertex $i \in V$, and the slot index $s$ has been dropped such that $\kappa^{*}$ represents the optimal scheduling for one slot. As finding MWIS is known to be NP-hard, there is no polynomial-time algorithm to solve $(\mathcal{P})$.

The evaluation of each vertex weight involves estimating the achievable data rate $r_{i}$. Since $r_{i}$ is a function of propagation path loss, inaccurate distance estimation may lead to suboptimal scheduling decisions. The assumption of perfect distance information can be relaxed by the discrete stochastic approximation, as follows: Let $\tilde{U}(\cdot)$ denote the noisy version of $U(\cdot)$, i.e., $\tilde{U}(\cdot)$ contains errors due to noisy distance information. Rewrite (11) as follows: $\kappa^{*}=\arg \max _{\kappa \in \mathcal{K}} \tilde{U}(\kappa)$, where $\tilde{U}(\kappa)=\sum_{i=1}^{|\kappa|} \tilde{U}\left(r_{i}\right)$. Subsequently, approximate $\tilde{U}(\kappa)$ by $\mathbb{E}\left[\tilde{U}_{m}(\kappa)\right]$, where $\mathbb{E}[\cdot]$ is the expectation operator, which yields

$$
\kappa^{*}=\arg \max _{\kappa \in \mathcal{K}} \sum_{i=1}^{|\kappa|} \tilde{U}\left(r_{i}\right) \approx \arg \max _{\kappa \in \mathcal{K}} \mathbb{E}[\tilde{U}(\kappa)] .
$$

If the objective function is unimodal, optimization techniques such as golden-section search or gradient-based approaches may be used to find the maxima of the nonlinear function $\mathbb{E}[\tilde{U}(\kappa)]$. In our consideration of heterogeneous traffic classes, however, the optimization objective function is not necessary to be unimodal. Furthermore, deriving the distribution of $\tilde{U}(\kappa)$ is very difficult, if not impossible, since $\tilde{U}(\kappa)$ is combinatorial, i.e., dependent on the element in $\kappa$. In situations where the

\footnotetext{
${ }^{1}$ An independent set in a graph is the set of vertices such that no two vertices in the independent set share the same edge. Associating each vertex with a weight, the independent set with the maximum total weight is the MWIS.
}

objective function is difficult to analytically derive, discrete approximation is an applicable technique to solve the optimization problems with uncertainties. Let $\left\{\tilde{U}_{m}(\kappa), m=1,2, \ldots\right\}$ represent the sequence of noisy utilities associated with set $\kappa \in \mathcal{K}$, where $\tilde{U}_{m}(\kappa)$ is obtained from different distance estimations. Furthermore, $\bar{U}(\kappa)=1 / M \sum_{m=1}^{M} \tilde{U}_{m}(\kappa)$ is the sample mean of $\{\tilde{U}(\kappa)\}$. By the strong law of large numbers, $\bar{U}(\kappa)$ almost surely (a.s.) converges to $\mathbb{E}[\tilde{U}(\kappa)]$. Together with the finiteness of the set $\mathcal{K}$, it is implied that

$$
\arg \max _{\kappa \in \mathcal{K}} \bar{U}(\kappa) \rightarrow \arg \max _{\kappa \in \mathcal{K}} \mathbb{E}[U(\kappa)] \quad \text { a.s. }
$$

Therefore, instead of using one biased utility value to solve the optimization problem $(\mathcal{P})$, we take the series $\left\{\tilde{U}_{m}(\kappa)\right\}$ in approximating the noisy objective function to avoid trapping into a local optimum [24].

\section{Proposed Algorithms}

In this section, we present a metaheuristic method, called the exclusive-region-based global search algorithm (ER-GSA), to efficiently find $\kappa^{*}$ in (13) for each time slot, followed by the algorithm for updating the utility value in each iteration.

\section{A. ER-GSA}

Intuitively, the optimal flow set $\kappa^{*}$ can be found by evaluating the utility value for each member in $\mathcal{K}$ to locate the maximal member. Such a brute force search is simple, but it cannot deal with estimation errors. In addition, the induced computation complexity exponentially grows as the network size increases, and most searching efforts are wasted on nonoptimal points in the solution space. Many metaheuristics, such as simulated annealing, Tabu search, genetic algorithm, and GSA [17], have been proposed to increase the search efficiency by defining "move sets" that allow the algorithm to move from one solution to superior solutions. In addition to the efficiency, the GSA is selected as the base to solve (13) since its convergence to a global optimum can be theoretically proved under certain conditions.

GSA relies on a random sequence generated during the algorithm iterations to efficiently find the optimum. The resulting random sequence is a Markov chain, where each state represents a point in the solution space that has been visited by the algorithm. In each iteration, the transition of the Markov chain is determined by comparing the objective value of the current state and that of a randomly chosen point from the solution space. We use the following notations in Algorithm 1: At the $m$ th iteration, $\kappa_{m}$ is the current state $\left(\kappa_{m} \in \mathcal{K}\right), W_{m}(\kappa)$ is the number of times the algorithm has visited state $\kappa$, and $\kappa_{m}^{*}$ is the state that is most frequently visited by the algorithm up to the $m$ th iteration.

\section{Algorithm 1: ER-GSA}

Step 1. Randomly select an initial user subset $\kappa_{0} \in \mathcal{K}$ and let $\kappa_{0}^{*}=\kappa_{0}$. Set $W_{0}\left(\kappa_{0}\right)=1$ and $W_{0}(\kappa)=0$ for all $\kappa \in \mathcal{K} \backslash\left\{\kappa_{0}\right\}$. Calculate $\bar{U}_{0}\left(\kappa_{0}\right)$. Let $m=0$ and go to step 2. 
Step 2. Randomly select another user subset $\kappa_{m}^{\prime} \in$ $\mathcal{K} \backslash\left\{\kappa_{m}\right\}$. Compute the corresponding $\bar{U}_{m}\left(\kappa_{m}^{\prime}\right)$ using the variable-sample method. Go to step 3.

Step 3. If $\bar{U}_{m}\left(\kappa_{m}\right)>\bar{U}_{m}\left(\kappa_{m}^{\prime}\right)$, let $\kappa_{m+1}=\kappa_{m}$ and go to step 5. Otherwise, go to step 4.

Step 4. Sort $\bar{U}_{m}\left(\kappa_{m}^{\prime}\right)$ in descending order. Denote $s_{i}$ the $i$ th flow in the sorted set and $S^{\prime}$ an empty set.

$$
\begin{aligned}
& \text { for } i=1 \text { to }\left|\kappa_{m}^{\prime}\right| \text { do } \\
& \quad \text { if } s_{i} \notin E R_{l} \& l \notin \\
& \quad S^{\prime}=S^{\prime} \cup\left\{s_{i}\right\} \\
& \text { end if } \\
& \text { end for }
\end{aligned}
$$

\section{end for}$$
\text { if } s_{i} \notin E R_{l} \& l \notin E R_{s_{i}} \forall l \in S^{\prime} \text { then }
$$

If $\bar{U}_{m}\left(S^{\prime}\right)>\bar{U}_{m}\left(\kappa_{m}^{\prime}\right)$, let $\kappa_{m}^{\prime}=S^{\prime}$. Let $\kappa_{m+1}=\kappa_{m}^{\prime}$ and go to step 5 .

Step 5. Let $m=m+1, W_{m}\left(\kappa_{m}\right)=W_{m-1}\left(\kappa_{m}\right)+1$, and $W_{m}(\kappa)=W_{m-1}(\kappa)$ for all $\kappa \in \mathcal{K} \backslash\left\{\kappa_{m}\right\}$. If $W_{m}$ $\left(\kappa_{m}\right)>W_{m}\left(\kappa_{m-1}^{*}\right)$, then let $\kappa_{m}^{*}=\kappa_{m}$. Otherwise, let $\kappa_{m}^{*}=\kappa_{m-1}^{*}$. Go to step 2 .

During iteration $m$, a new subset $\kappa_{m}^{\prime}$ is randomly selected (step 2), and the sample mean $\bar{U}_{m}\left(\kappa_{m}^{\prime}\right)=$ $1 /\left|\kappa_{m}^{\prime}\right|\left(\sum_{i=1}^{\left|\kappa_{m}^{\prime}\right|} \tilde{U}_{m}\left(\kappa_{i}\right)\right), \quad \kappa_{i} \in \kappa_{m}^{\prime}$ of the utility function value is compared with that of the current point $\kappa_{m}$ (step 3). $\bar{U}_{m}\left(\kappa_{m}^{\prime}\right)$ is calculated according to a variable-sample mean method (see Remark 2), with the number of sample equal to $\left|\kappa_{m}^{\prime}\right|$. The sequence $\left\{\kappa_{m}, m=1,2, \ldots\right\}$ is a Markov chain on the state space $\mathcal{K}$. If the current state is considered to be better than the newly selected state, i.e., $\bar{U}_{m}\left(\kappa_{m}\right)>\bar{U}_{m}\left(\kappa_{m}^{\prime}\right)$, the algorithm proceeds to step 5, updating the best subset $\kappa_{m}^{*}$ according to $W_{m}(\kappa)$. Since the optimal subset $\kappa_{m}^{*}$ has a higher probability of generating a larger utility function value, the associated $W_{m}\left(\kappa^{*}\right)$ is thus cumulated faster. To accelerate the convergence to the global optimum, a local enhancement in step 4 based on the concept of exclusive region is used to refine the chosen subset such that the optimal subset can be located faster. In particular, the set $E R_{l}$ contains those flows within the exclusive region of flow $l$, and $S_{i}$ denotes the $i$ th flow in $S$. The conditions $\left(S_{i} \notin E R_{l}\right)$ and $\left(l \notin E R_{S_{i}}\right)$ imply that flows $S_{i}$ and $l$ are allowed to concurrently transmit, according to (6). The size of exclusive region can be determined according to the strength of the background noise and the pathloss exponent [25], [26].

The GSA algorithm has some nice properties to allow its convergence. We apply these properties to prove the convergence of ER-GSA. We first state the convergence of ER-GSA.

Theorem 1 (Convergence of ER-GSA): Let $\mathcal{S} \in \mathcal{K}$ denote the set of global optimizers of the function $\tilde{U}(\kappa)$ (it is likely that multiple-user subsets can maximize the objective function). For each $i, j \in \mathcal{K}$, denote a random variable $Y^{(i \rightarrow j)}=\bar{U}(j)-$ $\bar{U}(i)$. If $Y^{(i \rightarrow j)}>0$, let $j$ be the next state. If $Y^{(i \rightarrow j)} \leq 0$, let $i$ be the next state. For all $\kappa^{*} \in \mathcal{S}, \kappa \notin \mathcal{S}$, and $\nu \in \mathcal{K} \backslash\left\{\kappa, \kappa^{*}\right\}$, if

C1) $\mathbb{P}\left\{Y^{\left(\kappa \rightarrow \kappa^{*}\right)}>0\right\}>\mathbb{P}\left\{Y^{\left(\kappa^{*} \rightarrow \kappa\right)}>0\right\}$.

C2) $\mathbb{P}\left\{Y^{\left(\nu \rightarrow \kappa^{*}\right)}>0\right\}>\mathbb{P}\left\{Y^{(\nu \rightarrow \kappa)}>0\right\}$.

Then, the sequence $\left\{\kappa_{m}^{*}\right\}$ a.s. converges to an element of $\mathcal{S}$.

Based on conditions $\mathrm{C} 1$ ) and $\mathrm{C} 2$ ), the algorithm convergence can be proved following [17, Th. 2.1]. Here, we verify these two conditions based on the following assumption.

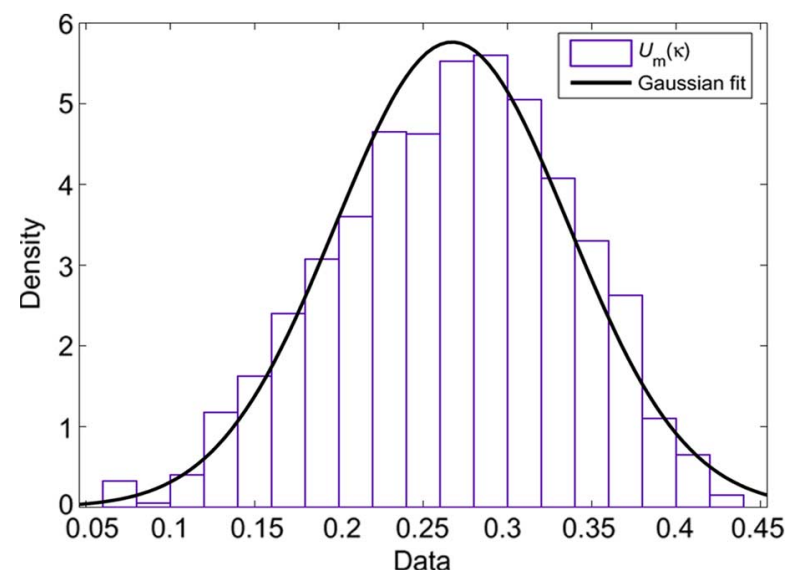

Fig. 3. Histogram of $\tilde{U}_{m}\left(\kappa_{m}\right)$ and the Gaussian fit.

Assumption 1: The estimation error of the distance between two devices is normally distributed. Then, $\tilde{U}(\kappa)$ as the sum of independent random variables (the estimated utilities of flows in subset $\kappa$ ) can be approximated as a Gaussian random variable.

Remark 1: Assumption 1 is given since the exact distribution of $\tilde{U}(\kappa)$ is difficult to obtain in practice. Simulation results verify that $\tilde{U}(\kappa)$ can be approximated by Gaussian distribution, as shown in Fig. 3, where the parameters are the same as those used in Section V.

Lemma 1: The Markov chain $\left\{\kappa_{m}\right\}$ is more likely to move from a nonoptimal state to an optimal state than the reverse direction, as stated in condition $\mathrm{C} 1)$.

Proof: Let $\tilde{U}(\kappa)$ and $\tilde{U}\left(\kappa^{*}\right)$ be Gaussian random variables with mean $\mathbb{E}[\tilde{U}(\kappa)]$ and $\mathbb{E}\left[\tilde{U}\left(\kappa^{*}\right)\right]$, respectively. Since $\kappa^{*} \in \mathcal{S}$ and $\kappa \notin \mathcal{S}, \mathbb{E}[\tilde{U}(\kappa)]<\mathbb{E}\left[\tilde{U}\left(\kappa^{*}\right)\right] . Y^{\left(\kappa \rightarrow \kappa^{*}\right)}$ and $Y^{\left(\kappa^{*} \rightarrow \kappa\right)}$ are Gaussian random variables with mean $\mathbb{E}\left[\tilde{U}\left(\kappa^{*}\right)\right]-\mathbb{E}[\tilde{U}(\kappa)]>$ 0 and $\mathbb{E}[\tilde{U}(\kappa)]-\mathbb{E}\left[\tilde{U}\left(\kappa^{*}\right)\right]<0$, respectively. Because of the symmetry of the Gaussian distribution, $\mathbb{P}\left\{Y^{\left(\kappa \rightarrow \kappa^{*}\right)}>0\right\}>0.5$ and $\mathbb{P}\left\{Y^{\left(\kappa^{*} \rightarrow \kappa\right)}>0\right\}<0.5$. Thus, condition C1) is satisfied.

Lemma 2: If the current state of the Markov chain is not an optimal state, the transition will be more likely made toward the optimal state than any other states, as stated in condition C2).

Proof: $\tilde{U}(\kappa)$ is a Gaussian random variable with variance equal to $|\kappa| \sigma^{2}=n \sigma^{2}$. The $n$-sample average of $\tilde{U}(\kappa)$, i.e., $\bar{U}(\kappa)$, is a Gaussian random variable with mean $\mathbb{E}[\tilde{U}(\kappa)]$ and variance equal to $\sigma^{2}$. Similarly, $\bar{U}\left(\kappa^{*}\right)$ and $\bar{U}(\nu)$ are Gaussian random variables with mean $\mathbb{E}\left[\tilde{U}\left(\kappa^{*}\right)\right]$ and $\mathbb{E}[\tilde{U}(\nu)]$, respectively, and their variances are equal to $\sigma^{2} . Y^{\left(\nu \rightarrow \kappa^{*}\right)}$ and $Y^{(\nu \rightarrow \kappa)}$ are Gaussian random variables with mean $\mathbb{E}\left[\tilde{U}\left(\kappa^{*}\right)\right]-\mathbb{E}[\tilde{U}(\nu)]$ and $\mathbb{E}[\tilde{U}(\kappa)]-\mathbb{E}[\tilde{U}(\nu)]$, respectively, and both of their variances are equal to $2 \sigma^{2}$. Since $\kappa^{*} \in \mathcal{S}$ and $\kappa \notin \mathcal{S}, \mathbb{E}\left[\tilde{U}\left(\kappa^{*}\right)\right]-$ $\mathbb{E}[\tilde{U}(\nu)]>\mathbb{E}[\tilde{U}(\kappa)]-\mathbb{E}[\tilde{U}(\nu)]$. Using the similar argument in the Proof of Lemma 1, $\mathbb{P}\left\{Y^{\left(\nu \rightarrow \kappa^{*}\right)}>0\right\}>\mathbb{P}\left\{Y^{(\nu \rightarrow \kappa)}>0\right\}$.

Remark 2: Condition $\mathrm{C} 2$ ) is fairly restrictive, and it may not hold using the original GSA. Since the estimated utilities of each flow are asymptotically independent Gaussian random variables with variance $\sigma^{2}$, we can satisfy condition C2) using a variable-sample method: In each iteration (step 2), if the selected subset $\kappa$ contains $|\kappa|=n$ flows, we take $n$ samples and calculate the sample mean $\bar{U}(\kappa)$ and let $Y^{(i \rightarrow j)}=\bar{U}(j)-$ $\bar{U}(i)$. Thus, the variance of $Y^{(i \rightarrow j)}$ is independent of the number of flows in the subsets. 


\section{B. Utility Update}

The utility update is designed such that the scheduling decision satisfies the long-term fairness criteria. We employ a simple rule based on weighted fair queuing [27], which normalizes the instantaneous utility of flow $i$ in slot $s$, denoted by $u_{i}(s)$, to the total utility that this flow has obtained, i.e., $\sum_{t=1}^{s-1} u_{i}(t)$. In addition, an individual user can be further discriminated, e.g., according to the price that he/she is charged for the bandwidth usage. To this end, we propose a control parameter, denoted as $\rho_{i}^{(s)}$, for flow $i$ in slot $s$, i.e.,

$$
\rho_{i}^{(s)}:=c_{i} /\left(\sum_{t=1}^{s-1} u_{i}(t)+\epsilon\right)^{s}
$$

where $c_{i}$ is a predefined parameter representing the revenue contribution or importance of a particular user, and $\epsilon>0$ is a small nominal constant to avoid a zero denominator. Consequently, the weighted utility for flow $i$ in slot $s$ is given by

$$
U_{i}=\rho_{i}^{(s)} u_{i}(s)
$$

where $u_{i}(s)$ is a function of throughput and traffic class corresponding to each flow, as discussed in the previous section. The scheduling policy (14) has the following properties: 1) The weighted utility as an exponential function of slot index $s$ ensures that the flows with less sum utility have a higher priority; 2) a flow is opportunistically scheduled if it has a higher utility value in the current slot than others; and 3) different levels of protection to traffic classes can be achieved by adjusting the parameter $c_{i}$.

\section{Performance Evaluation and FURTHER DISCUSSION}

Simulations are conducted to demonstrate the performance of the proposed utility-based scheduling algorithm. Two heuristic algorithms presented in [26], and TDMA transmissions are used as the benchmark. We start by tracking the instantaneous behavior of the proposed utility-based scheduling algorithm. We then use three metrics to study the long-term performance of our proposed solutions, namely the cumulative utilities, the minimum utility among flows, and the fairness support. Finally, the complexity and efficiency of the ER-GSA are evaluated.

\section{A. Experimental Setting}

The simulated network consists of 20 nodes uniformly distributed in a square area of $10 \times 10 \mathrm{~m}^{2}$. Each sender arbitrarily chooses another node as the receiver, forming ten peer-to-peer communication flows. The data rate of each flow is estimated as follows: $R=k \cdot W \log _{2}(1+\mathrm{SINR})$, where $0<k \leq 1$ reflects the efficiency of the transceiver design; $W=500 \mathrm{MHz}$; the UWB power spectrum densities of the transmission and noise are -41 and $-114 \mathrm{dBm} / \mathrm{MHz}$, respectively; and the pathloss exponent is set to 4 . The distance between two nodes $\tilde{d}$ is modeled by $d=\tilde{d}+\delta$, where $d$ is the actual distance, and $\delta$ is the estimation error approximated by a normal distributed
TABLE I

TRAFFIC CHARACTERISTICS FOR SIMULATION

\begin{tabular}{c|c|c}
\hline Traffic Class & B.W. Requirement & Utility Function \\
\hline \hline I & $1 \mathrm{Mbps}$ & $\begin{cases}1 & \text { if } r \geq 1, \\
0 & \text { if } r<1 .\end{cases}$ \\
\hline II & $1-20 \mathrm{Mbps}$ & $1-e^{\frac{-0.03 r^{2}}{20+r}}$ \\
\hline III & $0-250 \mathrm{Mbps}$ & $\begin{cases}1 & \text { if } r \geq 250, \\
\sin ^{0.3}\left(\frac{\pi}{2} \cdot \frac{r}{250}\right) & \text { if } r<250 .\end{cases}$ \\
\hline
\end{tabular}

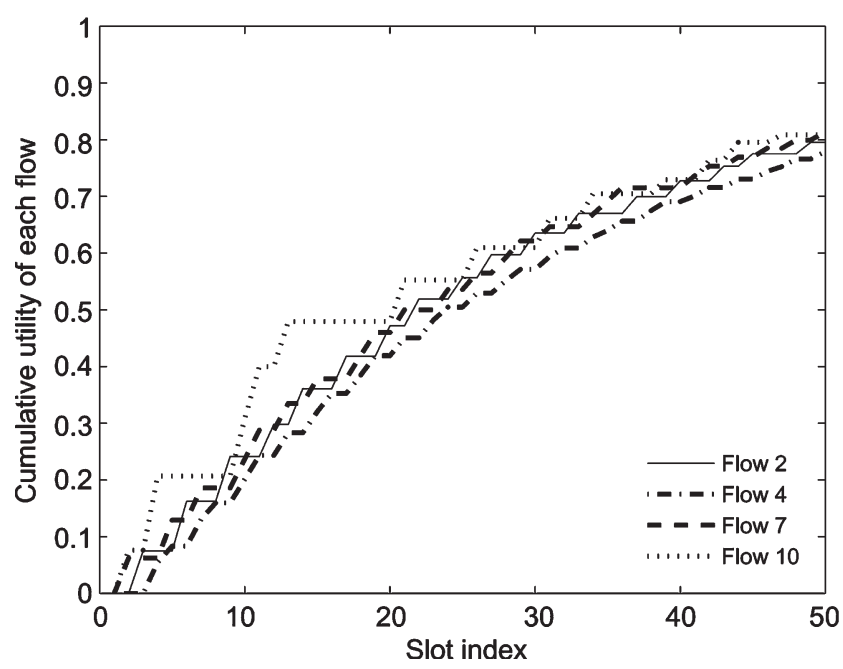

Fig. 4. Scheduling performance of the single-class case: ten Class- 3 flows. Four sample flows are shown with link distances of 5.73, 8.61, 6.93, and $3.89 \mathrm{~m}$, respectively.

random variable according to [28, eq. (15)], i.e., $\delta \sim \mathcal{N}\left(0, \sigma^{2}\right)$, where $\sigma^{2}=0.05$. The cross correlation of the target signal and the interfering signals is assumed to be 0.1 . Table I lists the considered three traffic classes and their utility functions. Each superframe contains ten slots. The size of exclusive region, which is denoted as $d_{E R}$, is set to $2 \mathrm{~m}$, except in Section V-C, where we vary the size of exclusive region to study its impact on the aforementioned three performance metrics.

\section{B. Utility-Based Scheduling}

The total utility that each flow has gained is defined as the cumulative flow utility, and we use two scenarios to demonstrate the long-term performance of the proposed utility-based scheduling. The first scenario consists of ten Class- 3 flows with a maximum bandwidth requirement of $250 \mathrm{Mb} / \mathrm{s}$. This corresponds to the situation where several high-bandwidth demanded users are active at the same time. Fig. 4 shows the cumulative flow utility. For this single traffic case, the equal utility should be ensured among users. Hence, the parameter $c_{i}$ in (14) is set to be 1 for all flows. In Fig. 4, a flat segment on the performance curve means that the flow is idle in that particular slot; the curve rises when a new slot is assigned. It is clear that each flow has a different scheduling pattern, but their cumulative utility, i.e., users' satisfactory level about the assigned bandwidth, is about the same in the long term. The effectiveness of the control parameter $\rho$ in (14) can also be inferred from the figure. As time elapses, the parameter $\rho$ of 


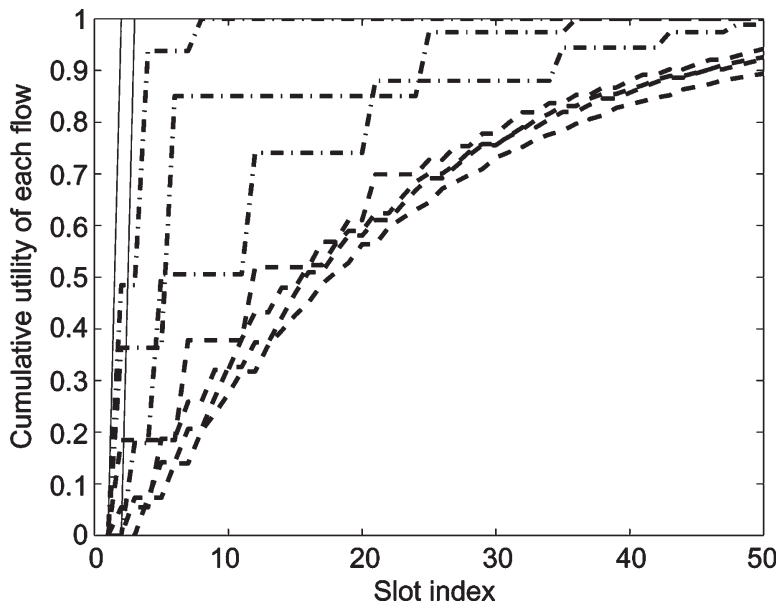

Fig. 5. Scheduling performance of the multiclass case. Three Class-1 flows (solid line), three Class-2 flows (dash-dot line), and four Class-3 flows (dashed line).

those users with more aggregate utilities are reduced, and thus, the users with less utility and a higher value of $\rho$ have a higher priority to be scheduled for transmissions in the subsequent slots.

The second scenario contains three traffic classes: 1) three Class-1 flows demanding a minimum bandwidth of $1 \mathrm{Mb} / \mathrm{s}$; 2) three Class-2 flows with a maximum bandwidth requirement of $20 \mathrm{Mb} / \mathrm{s}$; and 3) four Class-3 flows with a maximum bandwidth of $250 \mathrm{Mb} / \mathrm{s} . c_{i}$ 's are set to be $[2,10,1]$ for Class 1 , Class 2, and Class 3, respectively. Fig. 5 shows the cumulative flow utility in 50 slots. It can be seen that the flows within the same class achieve resembling utilities. Class-1 flows can achieve a cumulative utility of 1 in the first few slots because of their relatively lower bandwidth requirements and the use of step utility function.

\section{Utility versus Fairness}

An ideal scheduling algorithm should be able to maximize the total utility achieved in the network and guarantee fairness among competing flows. We compare the proposed scheduling algorithm with the two heuristic algorithms proposed in [26], i.e., the proportional allocation algorithm $(\mathrm{PaA})$ and the repeating allocation algorithm ( $\mathrm{RaA})$ (see the Appendix), since they employ the same concept of exclusive region to select the concurrent transmission set as ER-GSA does. Briefly, the PaA partition flows into several sets such that flows in the same set do not conflict with each other according to the exclusive region condition. The available slots are then allocated to these sets, where each set obtains a number of slots proportional to the number of flows within the set. On the other hand, RaA first picks the flow with the least number of assigned time slots and schedules as many flows in the same slot as possible, as long as each of these flows satisfies the rule of exclusive region. The same procedure is then repeated until all slots are allocated. Basically, $\mathrm{PaA}$ and $\mathrm{RaA}$ can guarantee a certain minimum utility with polynomial complexity but do not support fairness and differentiated services.

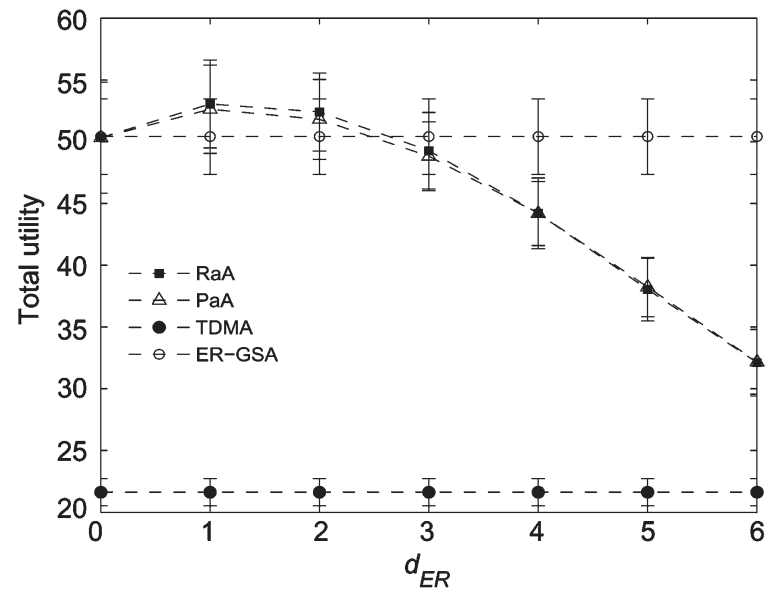

(a)

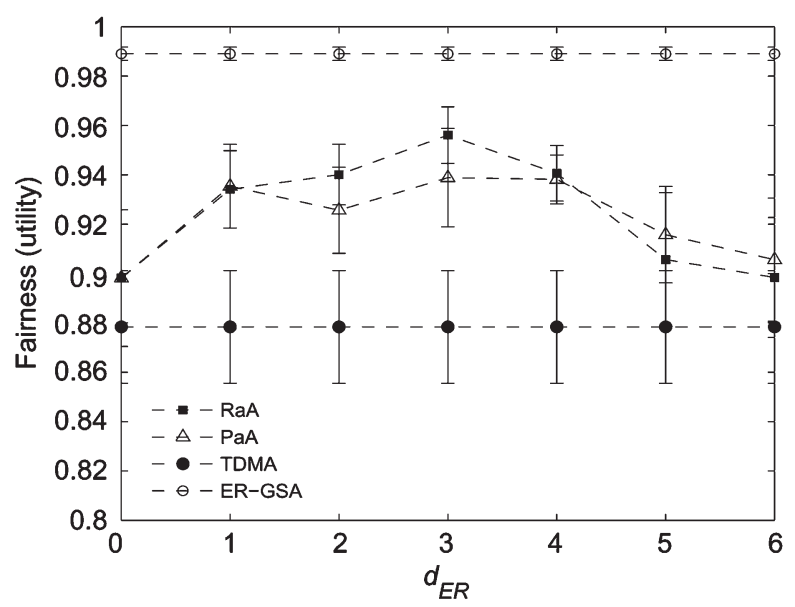

(b)

Fig. 6. Comparisons among different scheduling algorithms. One traffic class. (a) Total utility obtained by different scheduling algorithms. (b) Utility fairness obtained by different scheduling algorithms.

Three performance metrics are compared: 1) the total utility of all flows; 2) the minimum per-flow utility among all flows; and 3) Jain's fairness index [29]. Each point in Figs. 6-8 indicates the result at the end of the tenth slot. The $95 \%$ confidence interval from ten different random topologies is plotted as error bars. Other parameters follow the default setting defined at the beginning of this section.

1) Total Utility versus Fairness: Utility maximization and fair allocation are known to be two conflicting objectives. For instance, if we always choose the flows with better channel quality to transmit, we can achieve higher overall throughput and utility, with the consequence of starving some flows. Our proposed solution is to maximize the total utility under the fairness constraint. In Fig. 6(a), the total utility generated by ER-GSA, PaA, RaA, and TDMA among ten Class-3 flows are shown. For $\mathrm{PaA}$ and $\mathrm{RaA}$, the total utility significantly varies with respect to the size of exclusive region. ER-GSA achieves a comparable total utility as that achieved by $\mathrm{PaA}$ and $\mathrm{RaA}$ with the best $d_{E R}$. On the other hand, ER-GSA supports a much higher level of fairness than do $\mathrm{PaA}$ and $\mathrm{RaA}$, as shown in Fig. 6(b). TDMA maintains the best fairness in terms of the number of time slots allocated to each flow, but its achieved 


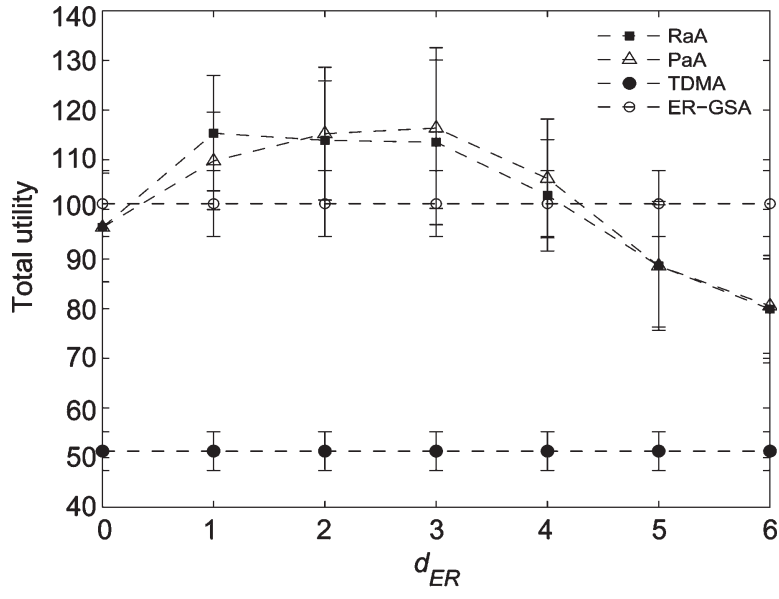

(a)

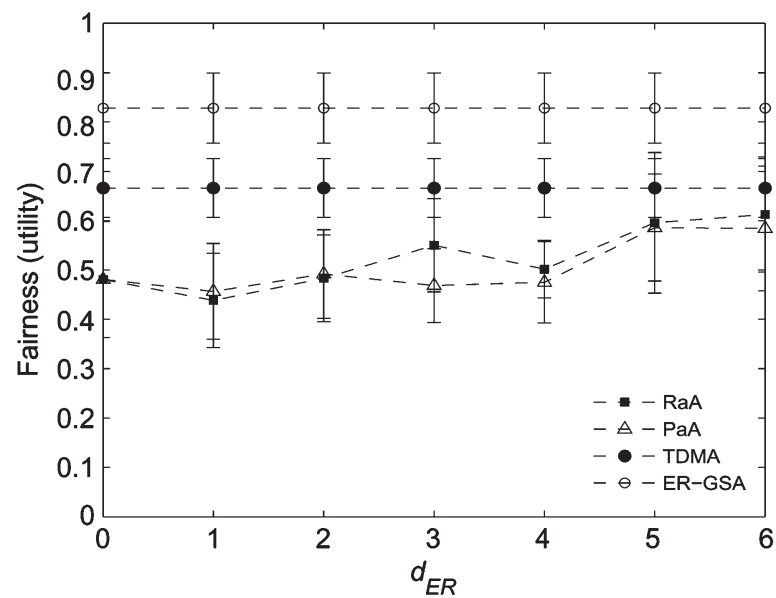

(b)

Fig. 7. Comparisons among different scheduling algorithms. Three traffic classes. (a) Total utility obtained by different scheduling algorithms. (b) Utility fairness obtained by different scheduling algorithms.

utility is much lower (about 58\% less) than that achieved by ER-GSA.

We further evaluate the performance of the case of three traffic classes using the same configuration as that in Section V-B. To measure fairness for heterogeneous traffic, Jain's fairness index is computed as $\sum_{i=1}^{N}(u(i) / c(i))^{2} /(N$. $\left.\sum_{i=1}^{N}(u(i) / c(i))^{2}\right)$, where $u(i)$ and $c(i)$ are the utility and the corresponding weighting factor of flow $i$, respectively. The total utility and the fairness index at the end of the tenth slot are shown in Fig. 7(a) and (b), respectively. For the total utility, ER-GSA achieves about $90 \%$ total utility of that achieved by $\mathrm{PaA}$ or RaA, but the latter two fail to maintain fairness in the presence of multiclass traffic. Together with the results in the single-class case, we conclude that ER-GSA can maintain a good balance between utility maximization and fairness.

2) Minimum Utility: According to the max-min fairness discipline, it is desirable to maximize the minimal per-flow utility among all competing flows. From Fig. 8(a) and (b), it can be seen that the achieved minimum utility using ER-GSA is much higher than that of other algorithms. Note that RaA can be considered as a max-min fair scheduler in terms of the number of time slots. In $\mathrm{RaA}$, the user with the minimum number of

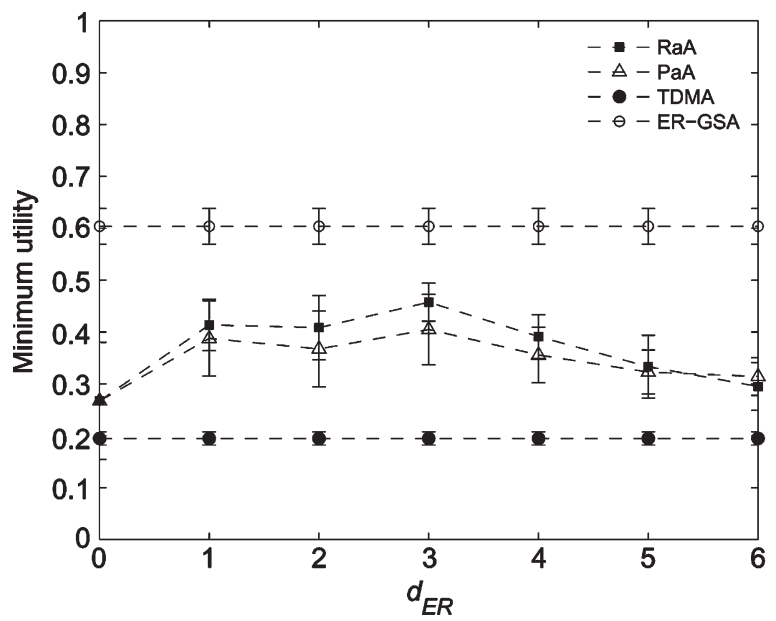

(a)

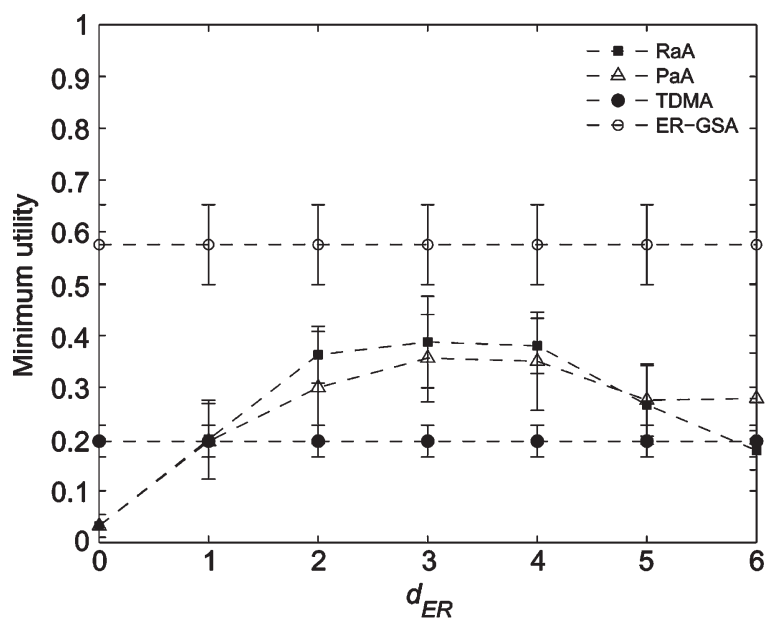

(b)

Fig. 8. Comparisons of the minimum utility. (a) One traffic class. (b) Three traffic classes.

assigned slots is chosen at each scheduling iteration. Thus, RaA performs slightly better than PaA by increasing the minimum utility. TDMA performs the worst.

\section{Algorithm Efficiency and Stability}

Finally, we study the computation efficiency and convergence issue of ER-GSA. Basically, ER-GSA converges in a statistical sense. In practice, a limited amount of time is allowed for executing the algorithm. To ensure that the algorithm promptly reaches the optimal point and steadily retains on the optimum, ER-GSA modifies the original GSA in two aspects: 1) In step 4, the strong interferers in the randomly selected flow set are further removed once a better set of flows is found, so that the resultant flow set is more likely to be the optimum, and 2) ER-GSA uses a variable-sample method in step 2 to ensure the convergence.

To evaluate the improvement in step 4, we compute the likelihood that the subset $S^{\prime}$ in step 4 in ER-GSA generates a higher utility than that by $\kappa_{m}^{\prime}$ in step 2, i.e., $\mathbb{P}\left[U_{m}\left(S^{\prime}\right)>\right.$ $\left.U_{m}\left(\kappa_{m}^{\prime}\right)\right]$. The amount of improvement depends on the network density and $d_{E R}$, as shown in Fig. 9. By increasing $d_{E R}$, more 


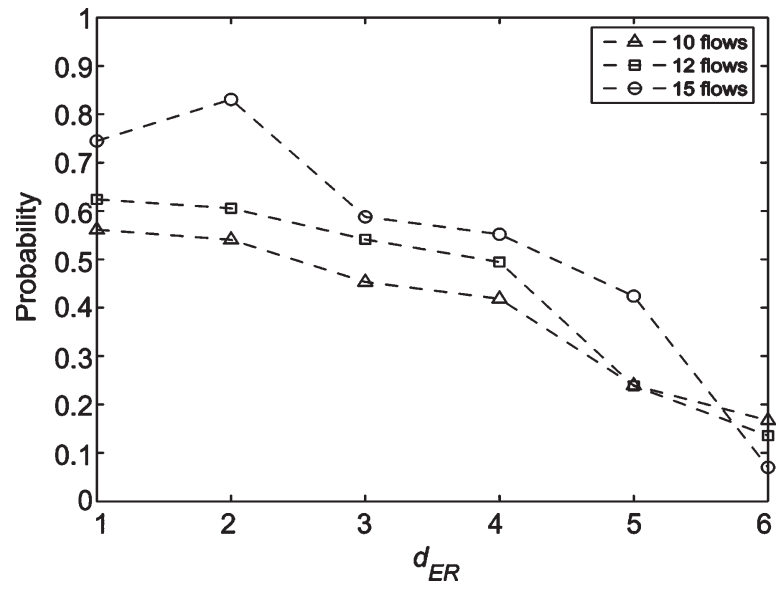

Fig. 9. $\mathbb{P}\left[U_{m}\left(S^{\prime}\right)>U_{m}\left(\kappa_{m}^{\prime}\right)\right]$ versus $d_{E R}$ in different network densities.

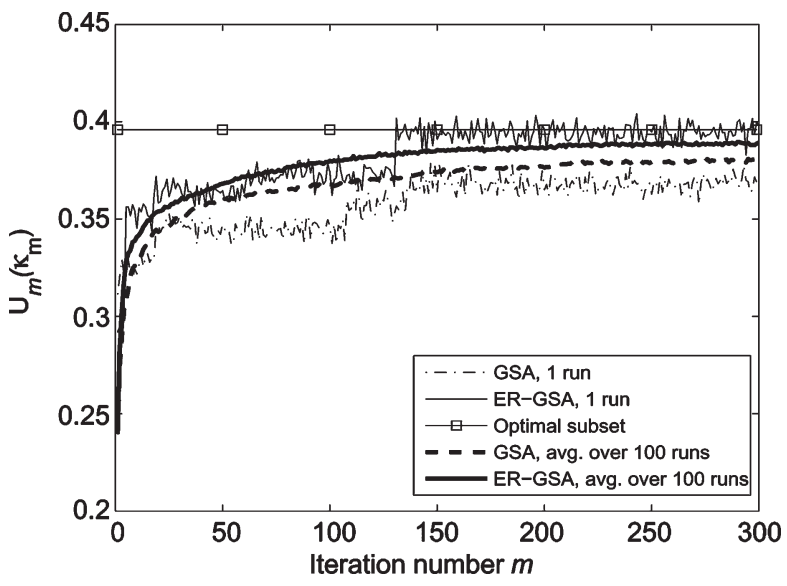

Fig. 10. Scheduling trajectory.

flows will be removed from $\kappa_{m}^{\prime}$, but the chance that $U_{m}\left(S^{\prime}\right)>$ $U_{m}\left(\kappa_{m}^{\prime}\right)$ is lower. Thus, a proper exclusive region size can ensure that $\mathbb{P}\left[U_{m}\left(S^{\prime}\right)>U_{m}\left(\kappa_{m}^{\prime}\right)\right]$ is larger than 0.5 such that the optimum may be located faster. The improvement is more significant when there are more flows to be scheduled.

Furthermore, Fig. 10 snapshots the utility achieved in a slot using GSA and ER-GSA with $d_{E R}=1 \mathrm{~m}$. The trajectory of a single run and that of the average of 100 runs are shown. The utility of the optimal set of flows is also plotted for comparison. The proposed ER-GSA can reach the optimal set with far fewer iterations than GSA.

On the other hand, it is also important that the algorithm can stay at the optimum state against measurement and estimation errors (noise), which is measured by the stability factor, as defined by the equation that follows. Denote $m_{i}$ and $m_{i}^{\prime}$ the number of iterations that the algorithm reaches and leaves the global optimum state at the $i$ th time, respectively. The stability factor $\xi$ of the searching algorithm is defined as

$$
\xi=\frac{\sum_{i}\left(m_{i}^{\prime}-m_{i}\right)}{M-m_{1}}
$$

where $\left(m_{i}^{\prime}-m_{i}\right)$ represents the number of iterations that the algorithm consecutively stays at the optimal point since the optimal point is reached at the $i$ th time, and $M$ is the total number of iterations. Generally, the smaller value of $\xi$ implies
TABLE II

EXECUTION TIME AND STABILITy $\xi$

\begin{tabular}{c||c|c|c|c|c}
\hline \hline Algorithm & GSA & ER-GSA & SPRS & RaA & PaA \\
\hline \multirow{5}{*}{$\begin{array}{c}\text { Execution } \\
\text { Time (ms) }\end{array}$} & 2.63 & 4.28 & 290.89 & 0.438 & 0.114 \\
\cline { 2 - 6 } & 2.61 & 3.44 & 362.06 & 0.560 & 0.110 \\
\cline { 2 - 6 } & 2.47 & 3.42 & 136.66 & 0.594 & 0.115 \\
\cline { 2 - 6 } & 2.58 & 3.43 & 206.73 & 0.706 & 0.078 \\
\cline { 2 - 6 } & 2.29 & 3.80 & 379.75 & 0.579 & 0.121 \\
\cline { 2 - 6 } & 2.66 & 3.52 & 261.06 & 0.589 & 0.076 \\
\cline { 2 - 6 } & 2.63 & 3.32 & 351.34 & 0.570 & 0.158 \\
\cline { 2 - 6 } & 2.58 & 3.30 & 325.87 & 0.595 & 0.122 \\
\cline { 2 - 6 } & 2.55 & 3.68 & 313.74 & 0.601 & 0.105 \\
\cline { 2 - 6 } & 2.71 & 3.34 & 309.51 & 0.593 & 0.106 \\
\hline Stability factor $\xi(\%)$ & 73 & 84 & 100 & - & - \\
\hline \hline
\end{tabular}

that the algorithm is more sensitive to disturbance. The impact of disturbance may be overcome by adaptively increasing the sample size, as suggested in the variable-sample path random search (SPRS) algorithm [30]. When the sample size grows sufficiently fast, the SPRS algorithm will stay at the optimal point once it is reached, at the cost of increased computation load.

We compare the stability and computation cost of all algorithms that are discussed. All algorithms are coded in $\mathrm{C}$ language and executed on a Pentium-IV 2.8-GHz central processing unit. We schedule ten flows for ten slots and repeat the algorithm for ten runs, where each run contains 1000 iterations. Table II shows the execution time and stability factor $\xi$ corresponding to each algorithm. It can be seen that SPRS has the best stability property, but it is not feasible to implement it for real-time scheduling. Considering the typical superframe length of 65-90 ms, the complexities of the other four algorithms are acceptable. Note that although the total computation time for the ER-GSA algorithm is slightly higher than that for the GSA algorithm, as shown in Fig. 9, ER-GSA has a better chance to converge to the optimum. In other words, if the algorithm is prematurely terminated due to insufficient computation time, the search result of ER-GSA is generally better. On the other hand, a sufficient number of iterations are needed to ensure the convergence of ER-GSA and GSA. A simple rule is to let it be at least the size of the entire solution space so that each point can be statistically checked once. More complicated termination rules, such as performing a paired $t$-test after a certain number of iterations to decide the termination (as in SPRS), may introduce excessive computation overhead, and thus, they are not recommended.

\section{RELATED WORK}

Using different spread-spectrum technologies, the UWB transmissions can be achieved by several approaches [31], [32]. In [18], the concept of exclusive region was proposed for UWB-based ad hoc networks. The study suggested that by implementing an exclusive region around each receiver, the optimal flow rate can be obtained, and the size of exclusive region impacts the achievable flow rate. The appropriate size of exclusive region was analyzed in [25] by taking power control into account and in [26] with fixed transmission power. Different from the previous work, in this paper, the concept of exclusive region is used to assist the proposed scheduling algorithm to quickly reach the optimum. 
To provide QoS in UWB networks, scheduling problems have also been considered in several recent studies. A max-min scheduling for centralized UWB networks was considered in [33] based on MB UWB. Another work for MB UWB was presented in [34]. Using the so-called MULTIFIT algorithm for binpacking problems, multiple-frequency bands over the UWB spectrum were allocated and scheduled to several WPANs in the vicinity. Such a joint channel allocation and servicedifferentiated scheduling scheme was shown to provide significant improvements in the throughput and packet delay via simulations. Different from the previous work where certain power control/allocation mechanisms were deployed to reduce power consumption and interference, our work focuses on deriving the optimal scheduling decision, whereas the processing load of PNC must be maintained as low as possible. Thus, we seek the solution when transmission power is fixed according to the Federal Communications Commission limit for unlicensed UWB transmissions. Although our strategy is not designed to reduce power consumption, our algorithm helps to ease the computation load of PNC, which may have a limited computation capability.

Furthermore, we focus on supporting heterogeneous traffic in UWB networks. A previous work [35] has proved that the optimal multiservice scheduling problem is NP-hard. Most existing works on multiservice scheduling are in fixed bandwidth allocation [8], [21]. In the context of concurrent peer-to-peer UWB networks, we further explore spatial multiplexing to improve the network throughput so that the scheduling problem is even more challenging.

\section{CONCLUSION}

In this paper, a utility-based optimal scheduling for concurrent UWB transmissions supporting heterogeneous traffic has been proposed. By defining an exclusive region around each receiver, several flows can be concurrently scheduled to increase the total utilities so that more high-bandwidth users can be accommodated. In addition, it is found that the size of the exclusive region in UWB networks is independent of the transceiver distance, which, on the contrary, is a dependent parameter in narrowband wireless systems. Simulation results have demonstrated that, for a single traffic class, our algorithm can provide $227 \%$ utility gain over TDMA. For multiple traffic classes, our algorithm can maximize the total utility under the constraints of both interclass and intraclass fairness. The proposed algorithm can also maintain a good balance between the computation complexity and the robustness against measurement and estimation errors, and thus, it suits UWB network schedulers with limited computation power.

\section{APPENDIX}

Algorithms 2-4, which were considered in Sections V-C and $\mathrm{D}$, are listed here for reference.

\section{Algorithm 2: SPRS [30]}

Step 1. Randomly select an initial user subset $\kappa_{0} \in \mathcal{K}$; $N_{0}:=$ initial sample size; $m:=0$. Go to step 2 .
Step 2. Given $\kappa_{m}$, calculate the corresponding $U_{m}\left(\kappa_{m}\right)$. Randomly select another user subset $\kappa_{m}^{\prime} \in \mathcal{K} \backslash\left\{\kappa_{m}\right\}$. Compute the corresponding $U_{m}\left(\kappa_{m}^{\prime}\right)$. Go to step 3 .

Step 3. If $U_{m}\left(\kappa_{m}\right)>U_{m}\left(\kappa_{m}^{\prime}\right)$, then let $\kappa_{m+1}=\kappa_{m}$. Otherwise, let $\kappa_{m+1}=\kappa_{m}^{\prime}$. Go to step 4 .

Step 4. Perform a paired $t$-test between $U_{m}\left(\kappa_{m}\right)$ and $U_{m}\left(\kappa_{m}^{\prime}\right)$ to test the null hypothesis, i.e., $H_{0}: U_{m}\left(\kappa_{m}\right)=$ $U_{m}\left(\kappa_{m}^{\prime}\right)$. If $p>0.2$, then $N_{m+1}:=N_{m}$; otherwise, $N_{m+1}:=$ $N_{m}+\delta_{N}$. For every $K$ iteration, do $N_{m+1}:=N_{m}+\delta_{N}$. $m:=m+1$. Go to step 2 .
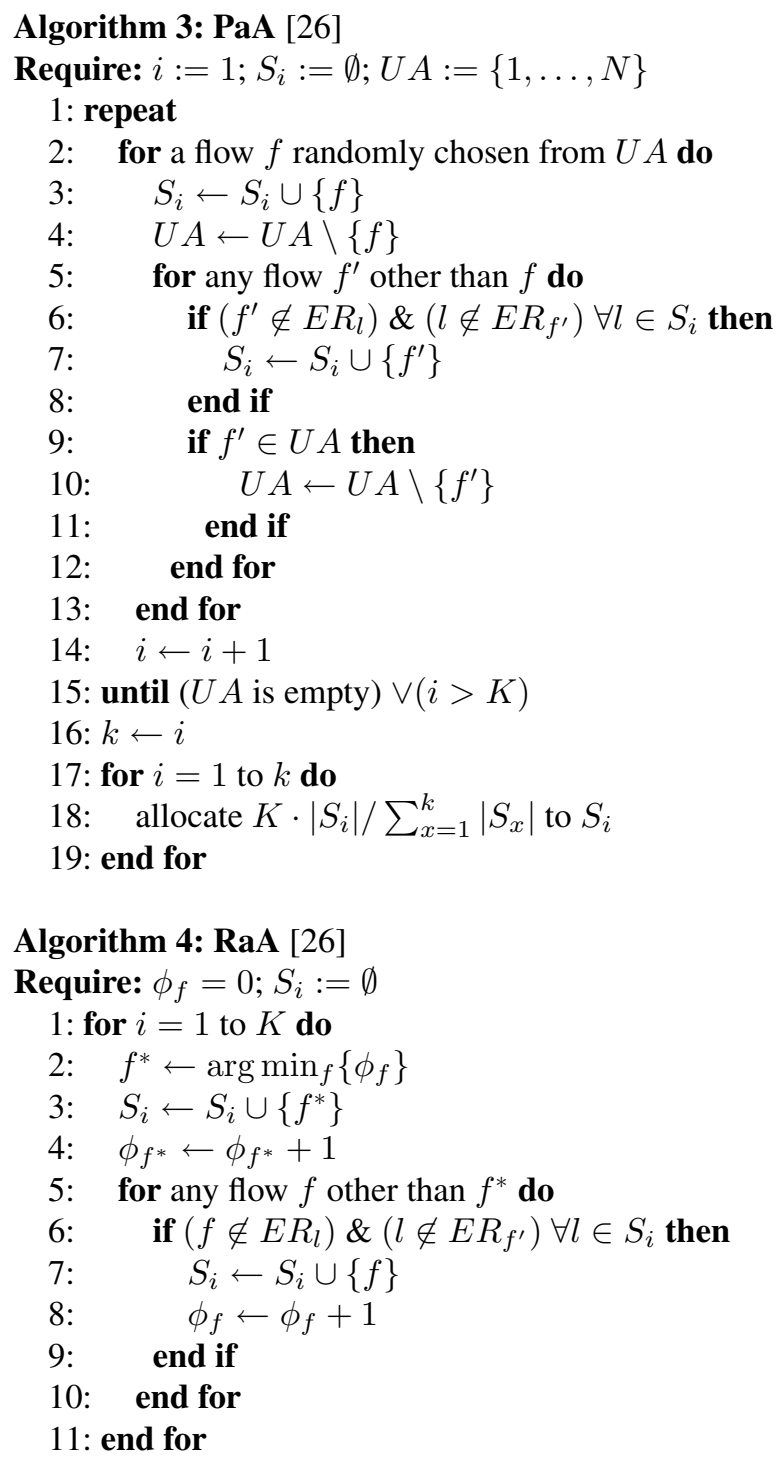

\section{ACKNOWLEDGMENT}

The authors would like to thank the anonymous reviewers for their valuable comments.

\section{REFERENCES}

[1] L. Yang and G. B. Giannakis, "Ultra-wideband communications: An idea whose time has come," IEEE Signal Process. Mag., vol. 21, no. 6, pp. 26-54, Nov. 2004.

[2] X. Shen, W. Zhuang, H. Jiang, and J. Cai, "Radio resource management for ultra-wideband communications," in Ultra-Wideband Wireless Communications and Networks. Hoboken, NJ: Wiley, 2006, pp. 189-209. 
[3] IEEE Standard Part 15.3: Wireless Medium Access Control (MAC) and Physical Layer (PHY) Specifications for High Rate Wireless Personal Area Networks WPANs, IEEE Std. 802.15.3-2003, Sep. 2003.

[4] IEEE 802.15 WPAN High Rate Alternative PHY Task Group 3a. DS-UWB Physical Layer Proposal, IEEE P802.15-04/0137r4, Jan. 2005.

[5] IEEE 802.15 WPAN High Rate Alternative PHY Task Group 3a. Multiband OFDM Physical Layer Proposal, IEEE P802.15-03/268r1, Sep. 2004.

[6] P. Gupta and P. R. Kumar, "The capacity of wireless networks," IEEE Trans. Inf. Theory, vol. 46, no. 2, pp. 388-404, Mar. 2000.

[7] Y. Cao and V. Li, "Utility-oriented adaptive QoS and bandwidth allocation in wireless networks," in Proc. IEEE ICC, New York, 2002, pp. 3071-3075.

[8] N. Lu and J. Bigham, "Utility-maximization bandwidth adaptation for multi-class traffic QoS provisioning in wireless networks," in Proc. 1st ACM Int. Workshop Q2SWinet, Montreal, ON, Canada, 2005, pp. 136-143.

[9] J. W. Lee, R. R. Mazumdar, and N. B. Shroff, "Downlink power allocation for multi-class DS-CDMA wireless networks," in Proc. IEEE INFOCOM, New York, 2002, pp. 1480-1489.

[10] M. Dianati, X. Shen, and S. Naik, "Cooperative fair scheduling for the downlink of CDMA cellular networks," IEEE Trans. Veh. Technol., vol. 56, no. 4, pp. 1749-1760, Jul. 2007.

[11] F. P. Kelly, A. K. Maulloo, and D. K. H. Tan, "Rate control in communication networks: Shadow prices, proportional fairness and stability," J. Oper. Res. Soc., vol. 49, no. 3, pp. 237-252, Mar 1998.

[12] C. E. Koksal, H. I. Kassab, and H. Balakrishnan, "An analysis of short-term fairness in wireless media access protocols," in Proc. ACM SIGMETRICS, Santa Clara, CA, Jun. 2000, pp. 118-119.

[13] L. Xu, X. Shen, and J. W. Mark, "Dynamic fair scheduling with QoS constraints in multimedia wideband CDMA cellular networks," IEEE Trans. Wireless Commun., vol. 3, no. 1, pp. 60-73, Jan. 2004

[14] D. B. Jourdan, J. J. Deyst, Jr., M. Z. Win, and N. Roy, "Monte Carlo localization in dense multipath environments using UWB ranging," in Proc. IEEE Conf. Ultra Wideband, Zurich, Switzerland, Sep. 2005, pp. 314-319.

[15] S. Gezici, Z. Tian, G. B. Giannakis, H. Kobayashi, A. F. Molisch, H. V. Poor, and Z. Sahinoglu, "Localization via ultra-wideband radios: A look at positioning aspects of future sensor networks," IEEE Signal Process. Mag., vol. 22, no. 4, pp. 70-84, Jul. 2005.

[16] J. C. Spall, Introduction to Stochastic Search and Optimization. New York: Wiley, 2003.

[17] S. Andradóttir, "A global search method for discrete stochastic optimization,” SIAM J. Optim., vol. 6, no. 6, pp. 513-530, May 1996.

[18] B. Radunovic and J.-Y. Le Boudec, "Optimal power control, scheduling, and routing in UWB networks," IEEE J. Sel. Areas Commun., vol. 22, no. 7, pp. 1252-1270, Sep. 2004.

[19] I. E. Telatar and D. N. C. Tse, "Capacity and mutual information of wideband multipath fading channels," IEEE Trans. Inf. Theory, vol. 46, no. 4, pp. 1384-1400, Jul. 2000

[20] F. Ramirez-Mireles, "On the performance of ultra-wide-band signals in Gaussian noise and dense multipath," IEEE Trans. Veh. Technol., vol. 50, no. 1, pp. 244-249, Jan. 2001.

[21] V. Rakocevic, J. Griffiths, and G. Cope, "Analysis of bandwidth allocation schemes in multiservice IP networks using utility functions," in Proc. 17th ITC, Salvador da Bahia, Brazil, Dec. 2001.

[22] J.-W. Lee, R. R. Mazumdar, and N. B. Shroff, "Non-convex optimization and rate control for multi-class services in the Internet," IEEE/ACM Trans. Netw., vol. 13, no. 4, pp. 827-840, Aug. 2005.

[23] A. Muqattash and M. Krunz, "CDMA-based MAC protocol for wireless ad hoc networks," in Proc. ACM MOBIHOC, Annapolis, MD, Jun. 2003, pp. $153-164$

[24] G. Pflug, Optimization of Stochastic Models. Norwell, MA: Kluwer, 1996.

[25] Y. Chu and A. Ganz, "A centralized MAC protocol for QoS support in UWB-based wireless networks," Wirel. Pers. Commun., vol. 34, no. 1, pp. 45-66, Jul. 2005.

[26] K.-H. Liu, L. Cai, and X. Shen, "Performance enhancement of medium access control for UWB WPAN," in Proc. IEEE Globecom, San Francisco, CA, Dec. 2006, pp. 1-5.

[27] A. Demers, S. Keshav, and S. Shenker, "Analysis and simulation of a fair queuing algorithm," in Proc. SIGCOMM, Austin, TX, 1989, pp. 1-12.

[28] B. Denis, J.-B. Pierrot, and C. Abou-Rjeily, "Joint distributed synchronization and positioning in UWB ad hoc networks using TOA," IEEE Trans. Microw. Theory Tech., vol. 54, no. 4, pp. 1896-1911, Apr. 2006.
[29] R. Jain, A. Durresi, and G. Babic, Throughput Fairness Index: An Explanation, Feb. 1999. ATM Forum Document Number: ATM Forum/990045.

[30] T. Homem-De-Mello, "Variable-sample methods for stochastic optimization," ACM Trans. Model. Comput. Simul. (TOMACS), vol. 13, no. 2 , pp. 108-133, Apr. 2003.

[31] M. Win and R. Scholtz, "Ultra-wide bandwidth time-hopping spreadspectrum impulse radio for wireless multiple-access communications," IEEE Trans. Commun., vol. 48, no. 4, pp. 679-689, Apr. 2000.

[32] V. S. Somayazulu, "Multiple access performance in UWB systems using time hopping vs. direct sequence spreading," in Proc. IEEE WCNC, Orlando, FL, Mar. 2002, pp. 522-525.

[33] A. Rajeswaran, G. Kin, and R. Negi, "A scheduling framework for UWB and cellular networks," Mob. Netw. Appl., vol. 11, no. 1, pp. 9-20, Feb. 2006.

[34] A. Rangnekar and K. M. Sivalingam, "QoS aware multi-channel scheduling for IEEE 802.15.3 networks," Mob. Netw. Appl., vol. 11, no. 1, pp. 47-62, Feb. 2006

[35] C. Lee, J. Lehoczky, R. Rajkumara, and D. Siewiorek, "On quality of service optimization with discrete QoS options," in Proc. 5th IEEE Real-Time Technol. Appl. Symp., Vancouver, BC, Canada, Jun. 1999, pp. 276-286.

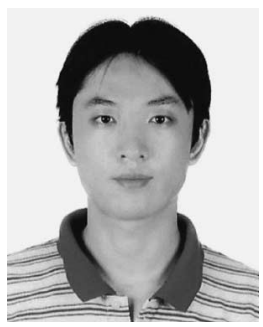

Kuang-Hao Liu received the B.S. degree in applied mathematics from National Chiao Tung University, Hsinchu, Taiwan, R.O.C., in 1998 and the M.S. degree in electrical engineering from National Chung Hsing University, Taichung, Taiwan, in 2000 . He is currently working toward the Ph.D. degree in electrical and computer engineering at the University of Waterloo, Waterloo, ON, Canada.

From 2000 to 2002, he was a Software Engineer with Siemens Telecommunication Systems Ltd. Hsinchu. His research interests include UWB communications in personal area networks, resource allocation problems, and performance analysis for wireless communication protocols.

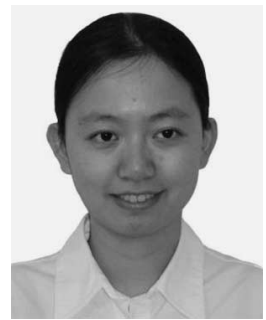

Lin Cai (S'00-M'06) received the M.A.Sc. and $\mathrm{Ph} . \mathrm{D}$. degrees in electrical and computer engineering from the University of Waterloo, Waterloo, ON Canada, in 2002 and 2005, respectively.

Since July 2005, she has been an Assistant Professor with the Department of Electrical and Computer Engineering, University of Victoria, Victoria, BC, Canada. Her research interests span several areas in wireless communications and networking, with a focus on network protocol and architecture design supporting emerging multimedia traffic over wireless, mobile, ad hoc, and sensor networks.

Dr. Cai is a member of the Association for Computing Machinery. She serves as an Associate Editor for the IEEE TRANSACTIONS ON VEHICULAR TECHNOLOGY, the EURASIP Journal on Wireless Communications and Networking, and the International Journal of Sensor Networks. She received an Outstanding Achievement in Gradute Studies Award while studying for the $\mathrm{Ph} . \mathrm{D}$. degree at the University of Waterloo. 


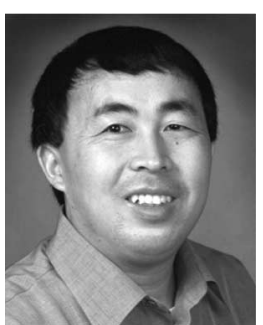

Xuemin (Sherman) Shen (M'97-SM'02) received the B.Sc. degree from Dalian Maritime University, Dalian, China, in 1982 and the M.Sc. and Ph.D. degrees from Rutgers University, Piscataway, NJ, in 1987 and 1990, respectively, all in electrical engineering. He is currently with the Department of Electrical and Computer Engineering, University of Waterloo, Waterloo, ON, Canada, where he is a Professor and the Associate Chair for Graduate Studies. $\mathrm{He}$ is the author or coauthor of more than 300 papers and book chapters on wireless communications and networks, control, and filtering and is a coauthor of three books. His research focuses on mobility and resource management in interconnected wireless/wired networks, UWB wireless communications systems, wireless security, and ad hoc and sensor networks.

Dr. Shen has served as the Technical Program Chair for many conferences, including the 2007 IEEE International Conference on Global Communications. He has also served as the Editor/Associate Editor/Guest Editor for the IEEE TRANSACTIONS ON WiRELESS COMMUNICATIONS, the IEEE TRANSACTIONS ON Vehicular TeChNOlogy, Computer Networks, ACM/Wireless Networks, the IEEE Journal on SELECTED AREAS IN TELECOMMUNiCATIONS, IEEE Wireless COMMUNICATIONS, IEEE Communications Magazine, etc. He was the recipient of the Outstanding Performance Award in 2002 and 2004 from the University of Waterloo for his outstanding contributions to teaching, scholarship, and service, as well as the Premier's Research Excellence Award in 2003 from the Province of Ontario, Canada, for the demonstrated excellence of his scientific and academic contributions. He is a Registered Professional Engineer in the Province of Ontario. 